\title{
Relaxation of abnormal rolls in planarly aligned electroconvection
}

\author{
S. Rudroff, V. Frette, and I. Rehberg \\ Institut für Experimentelle Physik der Universität Magdeburg, D-39016 Magdeburg, Germany
}

(Received 30 July 1998)

\begin{abstract}
Experimental results on electroconvection in a planarly aligned nematic liquid crystal are reported. Special emphasis is given to an optical technique for investigating abnormal rolls, which are characterized by a twist in the director field. The order parameter of the twist is measured by ellipsometry. We have also carried out a numerical investigation of the optical setup using a transfer-matrix method. The relaxation time of the twist has been measured and compared to theoretical predictions. In particular, it is found that the twist in the abnormal rolls forces the director field at the glass surface to turn away from its preferred direction (weak anchoring). This distortion in the anchoring of the liquid crystal relaxes one order of magnitude slower than theory predicts for relaxation processes driven by elastic forces in bulk. [S1063-651X(99)09701-9]

PACS number(s): 61.30.Eb, 47.65.+a, 42.70.Df, 42.30.Lr
\end{abstract}

\section{INTRODUCTION}

Electroconvection (EC) is a pattern-forming instability in a thin layer of a nematic liquid crystal [1,2]. A variety of roll patterns can be generated by applying an ac voltage across the sample. The most prominent patterns consist of normal rolls (NRs), where the roll axis is perpendicular to the director field. In addition, oblique rolls (ORs) can be observed in liquid crystals with a sufficiently low dielectric anisotropy. For increasing values of the driving voltage, these patterns become unstable. Zigzag and the skewed-varicose patterns are examples of roll configurations that arise as a result of such secondary, symmetry-breaking instabilities.

Recently, an intriguing new secondary instability in the director field has been found. This instability leads to abnormal rolls (ARs) characterized by a twist in the director field. The director's anchoring at the glass plates is expected to be parallel to the preferred direction ( $x$ axis), while in the bulk of the sample the director is twisted away from the $x$ axis. The twist angle $\varphi_{t}$ is maximal in the middle of the cell.

Two scenarios for generating abnormal rolls in the conductive regime of planarly aligned EC are possible [3]. For sufficiently high frequencies $\omega>\omega_{\mathrm{AR}}$, there is a direct transition from NRs to ARs. Below this frequency, oblique rolls are the precursor of abnormal rolls. The obliqueness of these rolls decreases continuously to zero when the voltage is increased, and at some point abnormal rolls stabilize [3,4].

In this paper, we present measurements of the direct transition from NRs to ARs, and of the relaxation dynamics of ARs. In Sec. II, we discuss the experimental setup and describe a measurement technique that is suitable for quantifying the twist of the ARs. The experimental results are presented in Sec. III.

\section{EXPERIMENTAL SETUP AND PROCEDURE}

The experimental setup is described in Ref. [5]. We observe electroconvection in the conductive regime of the liquid crystal 4-methoxygenzlidene- 4 ' $-n$-butylaniline (MBBA). We use planar alignment and perform the convection in a narrow channel parallel to the roll axis: a 25 $\times 10 \mathrm{~mm}$ ITO-coated glass plate faces another glass plate with an $L_{x}=315 \mu \mathrm{m}$ wide and $L_{y} \approx 10000 \mu \mathrm{m}$ long stripe of ITO. This stripe is manufactured by an etching process. The distance between the two plates is $d=24 \mu \mathrm{m}$. A mechanical treatment of the surfaces (rubbing) fixes the orientation of the director parallel to the glass plates. The rub direction defines the $x$ axis of the system and ensures that the normal rolls are parallel to the channel. During the measurements, the temperature of the experimental setup was 15 $\pm 1{ }^{\circ} \mathrm{C}$

The patterns are detected using a microscope and a CCDcamera. The standard shadowgraph technique is used to detect the roll pattern $[6,7]$. In order to measure the twist of the rolls, an ellipsometrical method is applied $[8,9]$. Here, we illuminate the convection cell with light which is polarized along the $y$ direction. Note that in this configuration normal rolls are not visible. In order to detect the ellipticity of the light, we use either a linear or a circular analyzer as shown in Fig. 1. The term "circular analyzer" denotes a combination of a $\lambda / 4$ plate and a linear polarizer and will be described in detail below.

In order to quantify the influence of the twist on the ellipticity of the light transmitted through the cell, we perform numerical calculations using the transfer-matrix formalism $[10,11]$. The light is represented by a two-dimensional vector $\vec{E}$ whose complex components give the amplitude and the phase of the electrical field. An optical component has a

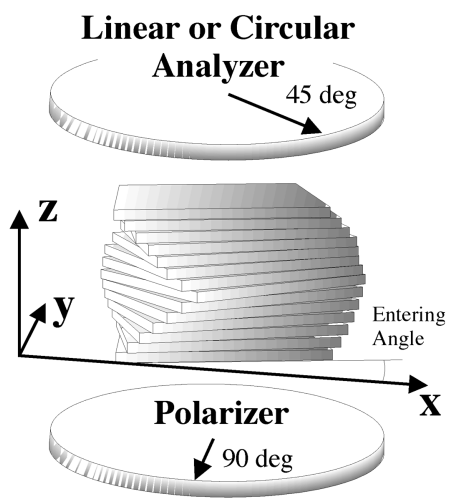

FIG. 1. Sketch of the optical setup. 
transfer matrix M, which gives its influence on $\vec{E}(t)$ through

$$
\vec{E}(t)=\left(\begin{array}{c}
0 \\
\cos (\omega t)+i \sin (\omega t)
\end{array}\right),
$$

with respect to the $x$ and the $y$ axes in the laboratory system. The intensity

$$
\frac{\omega}{2 \pi} \int_{0}^{2 \pi / \omega} \vec{E}(t)^{2} d t=\frac{\omega}{2 \pi} \int_{0}^{2 \pi / \omega}\left(E_{x} E_{x}^{*}+E_{y} E_{y}^{*}\right) d t
$$

of the incident light is thus equal to 1 . $\vec{E}^{\prime}(t)=\mathbf{M} \vec{E}(t)$. The incident light is given by

In order to apply the transfer-matrix method to the director field of a planarly aligned cell, we divide the liquidcrystal layer into $N=1000$ slices, enumerated by $j$, along the $z$ direction. We model the director of the $j$ th slice $(j$ $=0, \ldots, 999)$ by

$$
\varphi_{j}=\varphi_{t} \sin \left(\frac{\pi}{N} j\right)
$$

The angle $\varphi_{t}$ is the maximum twist angle. Then, for the $j$ th slice the transfer matrix is given by

$$
C=\frac{\frac{1}{\omega} \int_{0}^{2 \pi / \omega}\left(\vec{E} \times \frac{d \vec{E}}{d t}\right) \cdot \vec{e}_{z} d t}{\int_{0}^{2 \pi / \omega} \vec{E}^{2} d t}
$$

which is a measure for the area swept by the $E$ vector in one period. $\vec{e}_{z}$ is the unit vector in the $z$ direction. For perfect linearly polarized light, the circularity is zero. In the case of ideally circular polarized light, the circularity is +1 or -1 , depending on the direction of rotation of the $E$ vector.

Figure 3 shows the circularity of the light in a twisted layer of $24 \mu \mathrm{m}$ thickness as a function of the coordinate $z$. The maximum twist angle is $\varphi_{t}=20^{\circ}$. At $z=0$ the light is linearly polarized and its circularity is zero. As the light is propagating through the twisted nematic layer, the circularity has fast spatial oscillations with a length scale $\lambda_{c i}=\lambda /\left(n_{e}\right.$ $-n_{o}$ ) [10]. The fast oscillations are superimposed on a slowly varying modulation that seems to be connected with the twist. Note that the light leaving the cell has a finite circularity. The circularity is a function of the wavelength of the light, see Fig. 4. Here, the circularity of the transmitted light is shown for four different values of the twist angle. The solid line corresponds to $\varphi_{t}=20^{\circ}$, the angle used in Fig. 3.

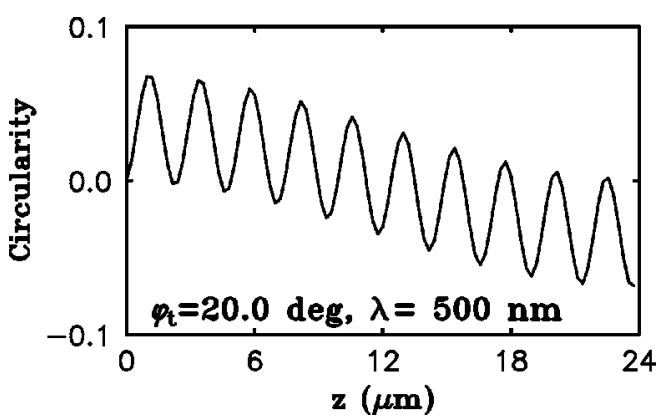

FIG. 2. Stereoscopic image: the trajectory of the $E$ vector for the light in 25 different planes normal to the $z$ axis in a twisted nematic

layer is shown $\left(d=4.5 \mu \mathrm{m}, \varphi_{t}=-20^{\circ}\right.$, and $\left.\lambda=500 \mathrm{~nm}\right)$.

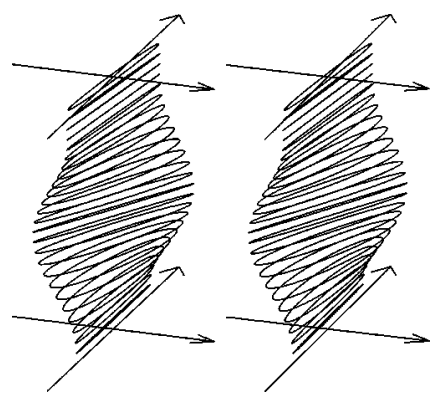

The standard measure for characterizing elliptically polarized light is the ellipticity, the ratio of the principal axes of the $E$-vector ellipse. This measure does not provide the diity" rection of rotation, however. We thus define the "circular-
FIG. 3. Circularity of light on propagating through a twisted nematic layer $\left(d=24 \mu \mathrm{m}, \varphi_{t}=20^{\circ}\right.$, and $\left.\lambda=500 \mathrm{~nm}\right)$. 


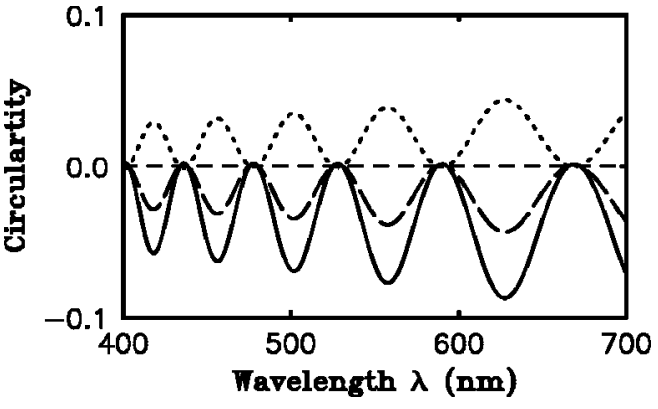

FIG. 4. Circularity of the transmitted light as a function of the wavelength $\lambda$ for a twist angle of $\varphi_{t}=-10^{\circ}$ (points), $0^{\circ}$ (dashes), $+10^{\circ}$ (long dashes), and $+20^{\circ}$ (solid line).

As can be seen from Fig. 4, the transmitted light will change its circularity with the twist angle and the wavelength of the propagating light. This circularity can be detected using either a linear analyzer (LA) or a circular analyzer (CA). In the LA configuration, a linear analyzer is placed between the convection cell and the CCD camera (see Fig. 1). This analyzer is set to $45^{\circ}$ with respect to the $x$ axis. In the CA configuration, the transmitted light first passes a $\lambda / 4$ plate, which is set to $45^{\circ}$ with respect to the $x$ axis. Then the light passes an additional linear analyzer, which is set to $90^{\circ}$ with respect to the $x$ axis. We denote this a configuration circular analyzer, even though, strictly speaking, it is the inversion of a circular polarizer.

We apply the transfer-matrix formalism also to the linear and the circular analyzer. For a linear analyzer at an angle $\alpha$ with respect to the $x$ axis, the transformation takes the form

$$
\vec{E}^{\prime}=\frac{1}{2}\left(\begin{array}{cc}
1+\cos 2 \alpha & \sin 2 \alpha \\
\sin 2 \alpha & 1-\cos 2 \alpha
\end{array}\right) \vec{E}
$$

and for a $\lambda / 4$ plate at an angle of $\gamma$ it reads

$$
\vec{E}^{\prime}=\frac{1}{2}\left(\begin{array}{cc}
\cos ^{2} \gamma+i \sin ^{2} \gamma & (1-i) \sin \gamma \cos \gamma \\
(1-i) \sin \gamma \cos \gamma & \sin ^{2} \gamma+i \cos ^{2} \gamma
\end{array}\right) \vec{E}
$$

Figure 5 shows the intensity after passage of the linear or circular analyzer $\left(\varphi_{t}=20^{\circ}\right)$. If there is no twist $\left(\varphi_{t}=0\right)$, $50 \%$ of the incident intensity is transmitted for both optical configurations. In the presence of a twist mode the transmitted intensity will depend on the wavelength of the incident light.

To determine the total intensity of the light that is detected by the CCD camera, one would have to take into

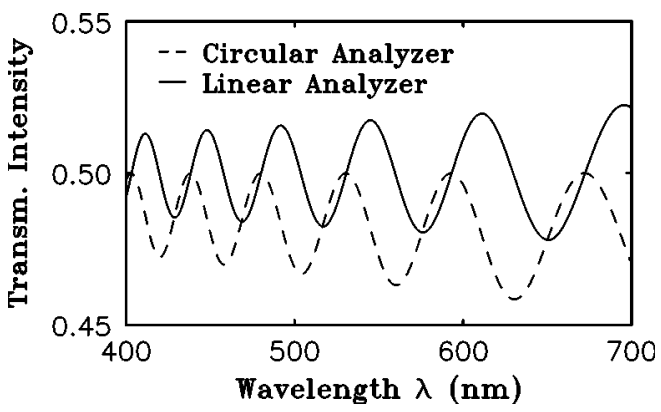

FIG. 5. Transmitted intensity for each of the optical configurations as a function of wavelength $\lambda$.

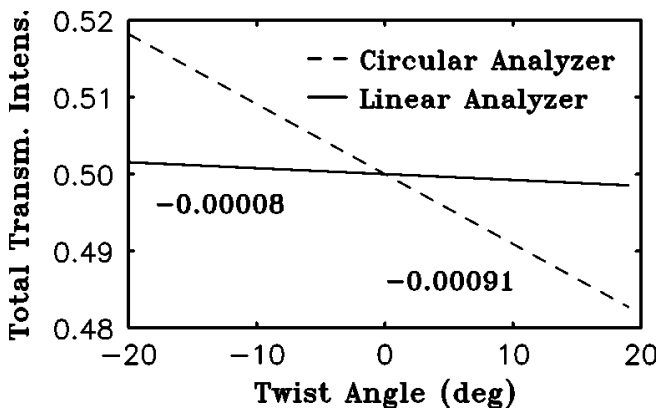

FIG. 6. Total transmitted intensity for each of the optical configurations as a function of the twist angle.

consideration the spectrum of the light source, the absorption spectrum of the nematic, and the spectral sensitivity of the CCD camera. To a first approximation, we neglect these effects and average the transmitted intensity from $400 \mathrm{~nm}$ to $675 \mathrm{~nm}$, since this corresponds to five oscillations in the transmitted intensity, as shown in Fig. 5. As mentioned above, the phase of these oscillations does not change with the twist angle $\varphi_{t}$.

In Fig. 6 we show the total transmitted intensity for both optical configurations as a function of the maximum twist angle $\varphi_{t}$. In both cases, the total transmitted intensity depends almost linearly on the twist angle $\varphi_{t}$ for $|\varphi|<20^{\circ}$. For the circular analyzer, the slope at $\varphi_{t}=0$ is $-0.00091 \mathrm{deg}^{-1}$, and for the linear analyzer it is $-0.00008 \mathrm{deg}^{-1}$. It is clear from Fig. 6 that the CA configuration displays a much stronger variation in the transmitted intensity when $\varphi_{t}$ varies than the LA configuration. From a measured intensity the maximum tilt angle may be obtained as

$$
\varphi_{t}=550^{\circ} \times \frac{I_{\mathrm{CA}}-I_{0}}{I_{0}}
$$

for the CA configuration and

$$
\varphi_{t}=6250^{\circ} \times \frac{I_{\mathrm{LA}}-I_{0}}{I_{0}}
$$

for the LA configuration. $I_{0}$ is the total transmitted intensity in the absence of a twist. Note that $I_{0}=0.5$ (see Fig. 6).

Thus, one may in principle choose between two optical setups for measuring a twist. However, we have demonstrated that the configuration with a circular analyzer is much more sensitive than the one with the linear analyzer.

For the calculations presented so far we have assumed the light to be polarized perpendicular to the director when entering the cell. In the experiment, this is not necessarily the case. The cell can either be misaligned with respect to the polarizer, or the liquid crystal might change its orientation at the glass plate due to weak anchoring [13]. Our procedure also allows examination of such a case. Results for an untwisted nematic layer are given in Fig. 7. Here, an entering angle of $0^{\circ}$ corresponds to light polarized perpendicular to the director. The figure demonstrates that the LA is much more sensitive to these finite entering angles than the CA. 


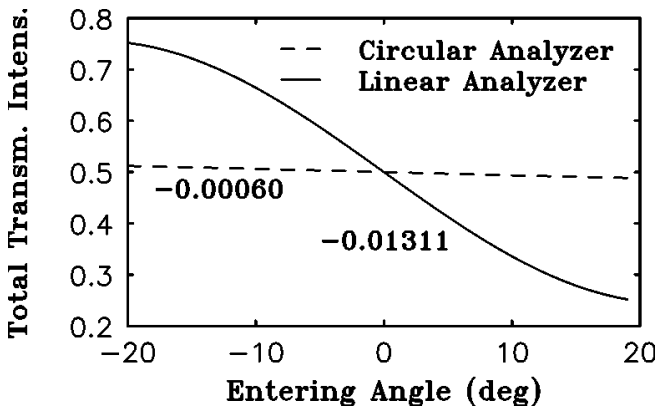

FIG. 7. Total transmitted intensity for each of the optical configurations as a function of the entering angle.

To summarize, the linear analyzer seems to be a sensitive tool for measuring entering angles, while the circular analyzer is more suitable for measuring a twist.

\section{EXPERIMENTAL RESULTS}

\section{A. Quasistatic measurements}

Figure 8 shows images of convection patterns in our cell for three different driving voltages at a frequency of $43 \mathrm{~Hz}$. In the upper part, the standard shadowgraph technique is used in order to visualize normal rolls (left), zigzag pattern (middle), and abnormal rolls (right). The lower parts show the same patterns using the ellipsometry with the CA configuration. The contrast has been enhanced by a factor of 10 . Small portions of these images are also displayed in the upper ones.
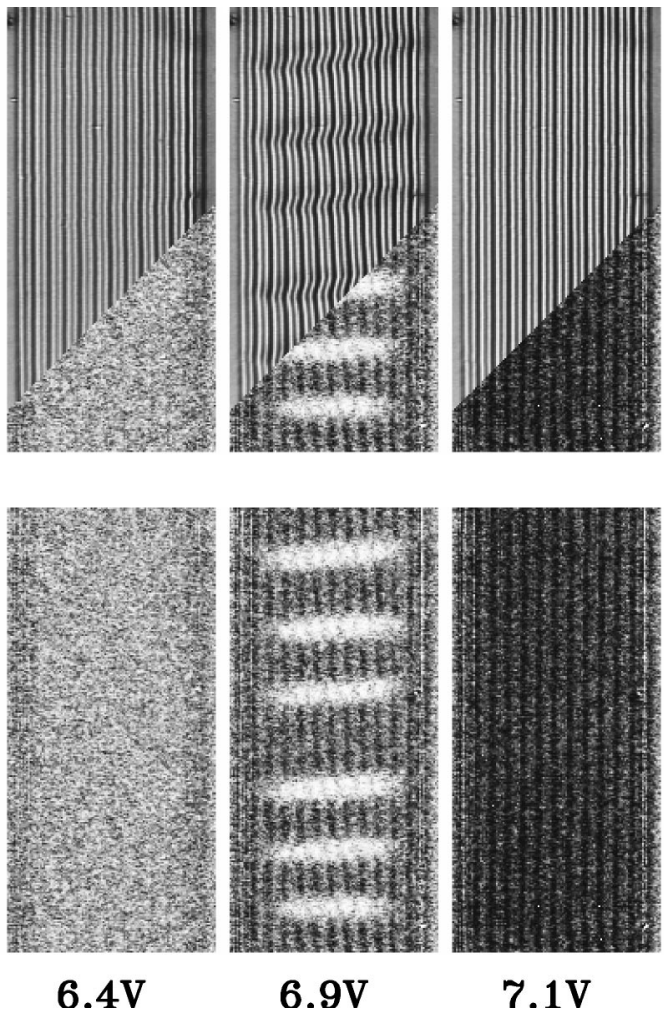

$7.1 \mathrm{~V}$

FIG. 8. Pattern obtained at three different voltages and a frequency of $43 \mathrm{~Hz}$. Upper parts: shadowgraph images; lower parts: CA images.
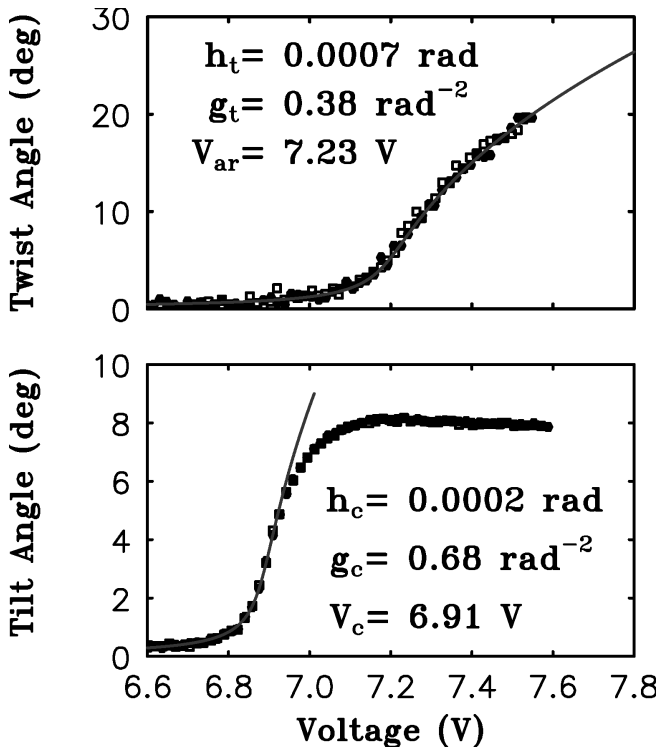

FIG. 9. Quasistatic measurements of the twist angle (upper part) and the tilt angle (lower part) at a frequency of $80 \mathrm{~Hz}$. Open squares correspond to increasing, solid circles to decreasing voltage. The solid lines are fits to the solution of an amplitude equation with a symmetry-breaking field.

The lower left-hand part in Fig. 8 demonstrates that the CA configuration is not suitable for visualizing normal rolls, because they have no twist. The gray level obtained in this configuration is used as a reference for $\varphi_{t}=0$. The middle part demonstrates nicely that zigzag patterns are accompanied by a twist with alternating sign, as indicated by the darker and lighter regions in the CA image. The shadowgraph image of the abnormal rolls (upper right-hand part) demonstrates the inability of this configuration to detect a twist. Twist does, however, manifest itself as a reduction of the mean intensity in the CA image. A slight modulation of the intensity along the $x$ axis is still visible. It is due to a spatial variation of the twist with the wave number of the abnormal rolls.

We now present quasistatic measurements of the direct normal-to-abnormal roll transition, which occur at higher frequencies $[3,4,14]$. At a fixed frequency of $80 \mathrm{~Hz}$, we increase the voltage from a subcritical value of $6.6 \mathrm{~V}$ to $7.6 \mathrm{~V}$, which is in the regime of abnormal rolls. This is done in 60 steps of the voltage. After each step, the system is allowed to relax for $60 \mathrm{~s}$. Upon reaching $7.6 \mathrm{~V}$, the voltage is decreased in the same manner to its initial value.

We make two independent measurements. First, we measure the intensity of the convection pattern using the shadowgraph method to detect the onset of convection [6]. The results for the director tilt angle are shown in the lower part of Fig. 9. The data indicate an imperfect bifurcation from the unstructured ground state to normal rolls, caused by the finite width of the cell [5]. The boundaries are forcing; convection rolls are more easily formed along the channel edges than in the interior of the system. This effect is very reminiscent of the imperfect bifurcation in a finite Taylor-Couette system [15]. We fit to the steady solution of an amplitude equation with a symmetry-breaking field,

$$
\tau_{c} \frac{d A}{d t}=\epsilon A-g_{c} A^{3}+h_{c} \quad \text { with } \quad \epsilon=\frac{V^{2}}{V_{c}^{2}}-1,
$$


where $A$ is the maximal tilt angle (strength of convection), $V_{c}$ gives the onset of convection, $\tau_{c}$ sets the temporal scale, $g_{c}$ is the equilibrium amplitude, and $h_{c}$ represents the symmetry breaking to lowest order. The value found for $g_{c}$ (see Fig. 9) corresponds to an angle (at $\epsilon=1$ ) of $70^{\circ}$, which should be compared to theoretical values that fall in the range $77^{\circ}-81^{\circ}$ [16]. However, the experiments were performed at a different temperature than assumed in the theory. An estimate for the strength of the symmetry breaking (rounding of the bifurcation) due to the finite system size can be obtained by comparing the tilt angle at $\epsilon=0$ (which is zero in an infinite system) to a characteristic tilt angle at $\epsilon$ $=1$. We find that $A(\epsilon=0) / A(\epsilon=1) \simeq h_{c}^{1 / 3} g_{c}^{1 / 6}=0.055$.

In a second measurement we determine the total transmitted intensity in the CA configuration. From this intensity, we calculate the maximum twist angle $\varphi_{t}$ by using Eq. (3). The result is shown in the upper part of Fig. 9. It indicates an imperfect bifurcation from normal to abnormal rolls. In the same manner as for the onset of convection, we fit the data to

$$
\tau_{t} \frac{d A}{d t}=\epsilon A-g_{t} A^{3}+h_{t} \quad \text { with } \quad \epsilon=\frac{V^{2}}{V_{\mathrm{AR}}^{2}}-1,
$$

where $A$ now represents the maximal twist angle as given by Eq. (3) and $V_{\text {AR }}$ gives the onset of abnormal rolls. The value of $g_{t}=0.38 \mathrm{rad}^{-2}$ can presently only be compared to a theoretical calculation by Plaut and Pesch [14]. They found $g_{t}$ $=0.67 \mathrm{rad}^{-2}$, but this value was obtained for a different nematic liquid crystal. The rounding of the bifurcation has a strength of $A(\epsilon=0) / A(\epsilon=1) \simeq h_{t}^{1 / 3} g_{t}^{1 / 6}=0.076$. However, this breaking of symmetry cannot be explained by the finite system size. A possible explanation could be that there is a small misalignment of the two plates that confine the sample. The imposed director directions at the two surfaces would then deviate slightly from being parallel, and, as a result, one of the two possible twist directions in the cell will be preferred (breaking of the symmetry).

As can be seen in Fig. 9, the amplitude of the pattern does not in any way indicate the direct transition from normal to abnormal rolls. Only the measurements with the CA configuration reveal this bifurcation.

\section{B. Dynamic measurements}

Now we present measurements of the relaxation of the abnormal rolls. We drive the system into the regime of abnormal rolls $(80 \mathrm{~Hz}, 7.8 \mathrm{~V})$ and let it relax for $120 \mathrm{~s}$. Then, we abruptly switch off the voltage and digitize one horizontal line of the pattern at a sampling rate of about $25 \mathrm{~Hz}$. This line covers not only the convection channel itself, it also contains a significant area outside the channel. We calculate the mean intensity of this part of the line, which is outside the convection channel. This gives a reference for the instantaneous intensity of the illumination at the moment when the line was digitized. We divide the whole digitized line by this value to get rid of possible fluctuations in the intensity of the light source.

To determine the relaxation of the director tilt angle $\Theta_{c}$ for the abnormal rolls, we approximate the director field by
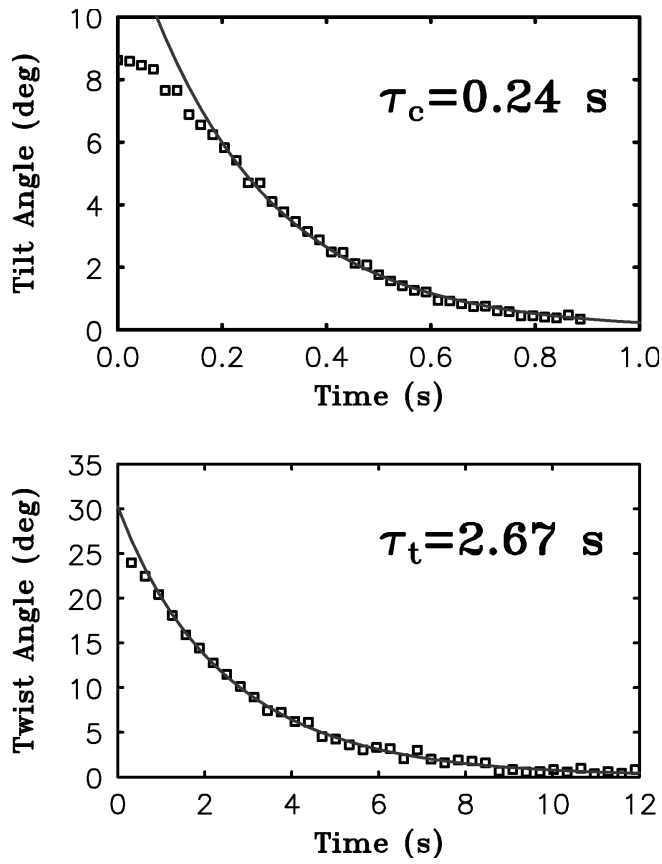

FIG. 10. Relaxation of abnormal rolls. The upper part shows the relaxation of the director tilt, and the lower part the relaxation of the twist angle, measured with the CA configuration.

$$
\hat{n}=\left(\begin{array}{c}
n_{x} \\
n_{y} \\
n_{z}
\end{array}\right)=\left(\begin{array}{c}
\Theta_{c} \cos (q x) \sin \left(\frac{\pi}{d} z\right) \\
0 \\
\Theta_{c} \sin (q x) \sin \left(\frac{\pi}{d} z\right)
\end{array}\right) .
$$

This approximation neglects the twist of the field, because we are using the shadowgraph method to analyze the tilt, and this method is not sensitive to a twist. An example of a shadowgraph image can be seen in the upper part of Fig. 8 . We calculate the power spectrum from the part of the digitized lines covering the six central convection rolls in the channel. From this spectrum we obtain the intensity $K$ of the peak that corresponds to the wave number $q=0.24 \mu \mathrm{m}^{-1}$. This intensity $K$ is for a sufficiently small tilt related to the maximum tilt angle via

$$
\Theta_{c}=0.61 K \frac{2 \pi}{q d} .
$$

The temporal evolution of $\Theta_{c}$ is given in the upper part of Fig. 10. The data are obtained by averaging over 50 relaxation processes. The solid line is a fitted exponential, and we obtain a relaxation time $\tau_{c}=0.24 \mathrm{~s}$ for the director tilt angle. A theoretical estimate for this relaxation time is

$$
\tau_{c}=\frac{\gamma_{1}}{k_{11}\left(\frac{\pi}{d}\right)^{2}+k_{33}(q)^{2}} .
$$

With the material parameters of MBBA [17] we get $\tau_{c}$ $=0.18 \mathrm{~s}$, in reasonable agreement with the measured value.

The lower part of Fig. 10 shows the relaxation of the twist of the abnormal rolls obtained with the CA configuration and 
using Eq. (8). The data are obtained by averaging over 50 measurements. We expect a relaxation process with a decay time

$$
\tau_{t}=\frac{\gamma_{1}}{k_{22}}\left(\frac{\pi}{d}\right)^{2}
$$

which is the linear relaxation time for a homogeneous twist in a cell of thickness $d$. With the material parameters of MBBA, we obtain $\tau_{t}=1.5 \mathrm{~s}$. The nonlinear relaxation of a pure, homogeneous twist mode is approximated by [18]

$$
\tau_{t} \frac{\partial}{\partial t} \varphi_{t}=-\varphi_{t}-\frac{1}{2} \varphi_{t}^{3}
$$

We use this simplified model to obtain an estimate for the relaxation in our more involved case, where both twist and tilt (convection) are present. It seems to be a reasonable assumption since convection rolls typically decay significantly faster than twist (see above). The solid line in Fig. 10 (lower part) is a fit by Eq. (16), and we obtain a relaxation time $\tau_{t}$ $=2.62 \mathrm{~s}$. The discrepancy with the theoretically expected value is presumably due to the fact that we are not dealing with a homogeneous state, which is also demonstrated on the right-hand side of Fig. 8. Note that the decay time $\tau_{t}$ is much longer than the one obtained for the tilt angle.

Finally, we would like to present measurements on the long-time behavior of the total transmitted intensity for both optical configurations. We measure the relaxation after switching off the voltage from the same value as in Fig. 10. The results are presented in Fig. 11. With the linear analyzer, one observes a relaxation on a time scale of $4 \mathrm{~min}$, as indicated by the solid line, which is a fit to an exponentially decaying function. With the CA configuration, the intensity relaxes with a decay time of $2.65 \mathrm{~s}$, which is indicated only by the first three data points within this plot.

A relaxation time of $4 \mathrm{~min}$ is much too large to be explained in terms of processes driven by the elastic forces in the liquid crystal. We assume that the twist causes the director to turn away from its initial orientation at the glass plates, a phenomenon called weak anchoring [13]. As argued in connection with Fig. 7, we expect the LA arrangement to be sensitive to changes in the entering angle. Thus, we propose that the long relaxation time is due to a reorientation of the director at the glass plates after the twist has vanished.
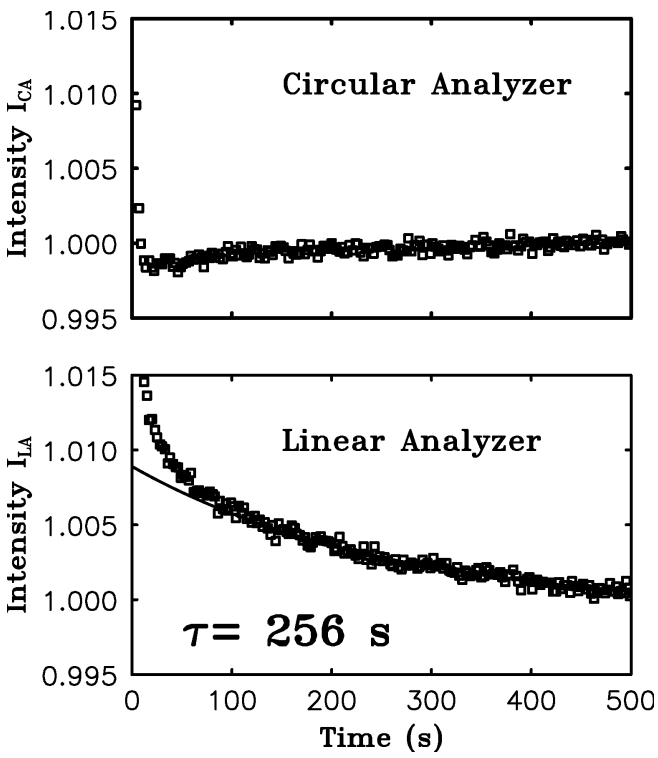

FIG. 11. Long-time relaxation in the CA and LA configurations.

\section{SUMMARY}

We have presented quantitative measurements on the transition from normal to abnormal rolls, and the relaxation dynamics of abnormal rolls. We have described two different optical configurations suitable for these measurements. Both methods were analyzed numerically. It was found that a circular analyzer is advantageous for measuring a twist, while a linear analyzer is more sensitive to a finite entering angle of the polarized light relative to the director. We believe these methods will be useful for further investigations of convection modes involving twist. Furthermore, the relaxation revealed an unexpected long-time behavior. This might be connected with a reorientation of the director at the glass plates, but this effect needs further quantitative investigation.

\section{ACKNOWLEDGMENTS}

It is a pleasure to thank A. Buka, N. Eber, W. Pesch, E. Plaut, and especially M. Grigutsch, and R. Stannarius for stimulating and clarifying discussions. V.F. has been supported by the TMR Program "Patterns, Noise and Chaos" of the EU. The experiments have been supported by NATO Grant No. CRG 950243 and Deutsche Forschungsgemeinschaft Grant No. Re 588/12-1.
[1] A. Joets and R. Ribotta, J. Phys. (Paris) 47, 595 (1986).

[2] I. Rehberg, B. L. Winkler, M. de la Torre Juarez, S. Rasenat, and W. Schöpf, Adv. Solid State Phys. 29, 35 (1989).

[3] E. Plaut, W. Decker, W. Pesch, A. Rossberg, L. Kramer, A. Belaidi, and R. Ribotta, Phys. Rev. Lett. 79, 2367 (1997).

[4] S. Rudroff, H. Zhao, L. Kramer, and I. Rehberg, Phys. Rev. Lett. 81, 4144 (1998).

[5] S. Rudroff and I. Rehberg, Phys. Rev. E 55, 2742 (1997).

[6] I. Rehberg, F. Hörner, and G. Hartung, J. Stat. Phys. 64, 1017 (1991).

[7] A. Joets and R. Ribotta, J. Phys. I 4, 1013 (1994).

[8] M. Grigutsch, N. Klöpper, H. Schmiedel, and R. Stannarius, Mol. Cryst. Liq. Cryst. Sci. Technol., Sect. A 262, 283 (1995).
[9] H. Amm, M. Grigutsch, and R. Stannarius, Mol. Cryst. Liq. Cryst., Sect. A (to be published); H. Amm, R. Stannarius, and A. G. Rossberg (unpublished).

[10] T. J. Scheffer and J. Nehring, J. Appl. Phys. 56, 908 (1984).

[11] A. Röseler, Infrared Spectroscopic Ellipsometry (AkademieVerlag, Berlin, 1990).

[12] C. Mauguin, Bull. Soc. Fr. Mineral 34, 71 (1911).

[13] B. Jerome, Rep. Prog. Phys. 54, 391 (1991).

[14] E. Plaut and W. Pesch (unpublished).

[15] G. Pfister and I. Rehberg, Phys. Lett. 83A, 19 (1981).

[16] E. Bodenschatz, W. Zimmermann, and L. Kramer, J. Phys. (France) 49, 1875 (1988). 
[17] At room temperature, MBBA has approximately elastic constants $\left(k_{11}, k_{22}, k_{33}\right)=(6.66,4.20,8.61) \times 10^{-12} \mathrm{~N}$, rotational viscosity $\gamma_{1}=0.1093 \mathrm{~kg} \mathrm{~m}^{-1} \mathrm{~s}^{-1}$, dielectric constants $\left(\epsilon_{\|}, \epsilon_{\perp}\right)=(4.72,5.25), \quad$ refraction indices $\left(n_{\|}, n_{\perp}\right)$ $=(1.75,1.54), \quad$ electric conductivity $\quad\left(\sigma_{\|}, \sigma_{\perp}\right)=(1.5 .1)$ $\times 10^{-7} \Omega^{-1} \mathrm{~m}^{-1}$. The dielectric constant is $\epsilon_{0}=8.85$ $\times 10^{-12} \mathrm{~s}^{4} \mathrm{~A}^{2} \mathrm{~kg}^{-1} \mathrm{~m}^{-3}$. This parameters are taken from Ref. [14].

[18] L. M. Blinov, Electro-optical and Magneto-optical Properties of Liquid Crystals (Wiley, Chichester, 1983). 